\title{
Is watch and wait a safe and effective way to treat rectal cancer in older patients?
}

Citation for published version (APA):

Haak, H. E., Maas, M., Lambregts, D. M. J., Beets-Tan, R. G. H., Beets, G. L., \& Dutch Watch-and-Wait Consortium (2020). Is watch and wait a safe and effective way to treat rectal cancer in older patients? European Journal of Surgical Oncology, 46(3), 358-362. https://doi.org/10.1016/j.ejso.2020.01.005

Document status and date:

Published: 01/03/2020

DOI:

10.1016/j.ejso.2020.01.005

Document Version:

Publisher's PDF, also known as Version of record

\section{Document license:}

Taverne

\section{Please check the document version of this publication:}

- A submitted manuscript is the version of the article upon submission and before peer-review. There can be important differences between the submitted version and the official published version of record.

People interested in the research are advised to contact the author for the final version of the publication, or visit the DOI to the publisher's website.

- The final author version and the galley proof are versions of the publication after peer review.

- The final published version features the final layout of the paper including the volume, issue and page numbers.

Link to publication

\footnotetext{
General rights Owners
rights.

- You may freely distribute the URL identifying the publication in the public portal. please follow below link for the End User Agreement:

www.umlib.nl/taverne-license

Take down policy

If you believe that this document breaches copyright please contact us at:

repository@maastrichtuniversity.nl

providing details and we will investigate your claim.
}

Copyright and moral rights for the publications made accessible in the public portal are retained by the authors and/or other copyright owners and it is a condition of accessing publications that users recognise and abide by the legal requirements associated with these

- Users may download and print one copy of any publication from the public portal for the purpose of private study or research.

- You may not further distribute the material or use it for any profit-making activity or commercial gain

If the publication is distributed under the terms of Article $25 \mathrm{fa}$ of the Dutch Copyright Act, indicated by the "Taverne" license above, 


\title{
Is watch and wait a safe and effective way to treat rectal cancer in older patients?
}

\author{
Hester E. Haak ${ }^{\text {a, c }}$, Monique Maas ${ }^{\text {b }}$, Doenja M.J. Lambregts ${ }^{\text {b }}$, Regina G.H. Beets-Tan ${ }^{\text {b. c }}$, \\ Geerard L. Beets ${ }^{a, c, *}$, on behalf of the Dutch Watch-and-Wait Consortium \\ a Department of Surgery, Netherlands Cancer Institute - Antoni van Leeuwenhoek, Amsterdam, the Netherlands \\ b Department of Radiology, Netherlands Cancer Institute - Antoni van Leeuwenhoek, Amsterdam, The Netherlands \\ ${ }^{\mathrm{c}}$ GROW School for Oncology and Developmental Biology - Maastricht University, Maastricht, the Netherlands
}

\section{A R T I C L E I N F O}

\section{Article history:}

Received 15 November 2019

Received in revised form

13 December 2019

Accepted 3 January 2020

Available online 8 January 2020

\section{Keywords:}

Rectal cancer

Chemoradiation

Watch-and-wait approach

Older patients

Oncological outcomes

Functional outcomes

\begin{abstract}
A B S T R A C T
Introduction: The aim was assess the oncological and functional outcome of the watch-and-wait (W\&W) approach in older patients with a clinical (near)complete response after neoadjuvant treatment for rectal cancer.

Material and methods: Patients were included in a W\&W-approach (2004-2019) when digital rectal examination, endoscopy and MRI showed a (near)clinical complete response. Patients underwent endoscopy and MRI every 3 months during the first year, and 6-monthly thereafter. Patients aged $\geq 75$ and $\geq 2$ years of follow-up (FU) were selected. Oncological outcomes were assessed with Kaplan-Meier curves. Functional outcome was assessed with colostomy-free rate, Vaizey incontinence score, low anterior resection syndrome-score and International Prostate Syndrome Score.

Results: 43/304 (14\%) of patients in a W\&W-approach met the inclusion criteria. Median FU was 37 (24 $-109)$ months. 5/43(12\%) developed a local regrowth. All were treated surgically, with one patient experiencing a pelvic failure. Distant metastases occurred in 3/43 patients and 4 patients died, 3 of whom not related to rectal cancer. The 3-year local regrowth-free rate was 88\%, 3-year non-regrowth diseasefree survival $91 \%$, overall survival $97 \%$ and 3 -year colostomy-free rate $93 \%$. Overall, the bowel- and urinary dysfunction scores at 3,12 and 24 months indicated good continence, no or minor LARS and moderate urinary problems.

Conclusion: $\mathrm{W} \& \mathrm{~W}$ for older patients with a clinical (near) complete response appears to be a safe alternative to a total mesorectal excision (TME), with a very high pelvic control rate, and few rectal cancer related deaths. Most patients can avoid major surgery and a definitive colostomy, and have a reasonable anorectal and urinary function.
\end{abstract}

๑ 2020 Elsevier Ltd, BASO The Association for Cancer Surgery, and the European Society of Surgical Oncology. All rights reserved.

\section{Introduction}

All patients with rectal cancer are faced with important questions regarding treatment options and outcome that are related to anorectal and urogenital dysfunction, the possibility of a permanent stoma, and the balance between quality of life and oncological

\footnotetext{
* Corresponding author.The Netherlands Cancer Institute - Antoni van Leeuwenhoek, Department of Surgery, Postbox 90203, 1006, BE, Amsterdam, the Netherlands.

E-mail addresses: h.haak@nki.nl (H.E. Haak), m.maas@nki.nl (M. Maas), d. lambregts@nki.nl (D.M.J. Lambregts), r.beetstan@nki.nl (R.G.H. Beets-Tan), g. beets@nki.nl (G.L. Beets).
}

control. Older patients have additional concerns of operative morbidity and mortality, and the loss of independency that can occur after major rectal cancer surgery. Age by itself is not a good predictor of operative outcome, and there are many tools to assess frailty and the operative risk [1]. At the far end of the spectrum is the very frail patient who clearly cannot tolerate a major rectal resection. However, for the majority of older patients who have some degree of elevated operative risk, major rectal surgery is still an option if the alternative of local control by radiotherapy fails. With a good screening program for frailty and appropriate preoperative, perioperative and postoperative care the mortality rate can be much lower than traditionally estimated. Recent data from a national Dutch registry shows a marked improvement in the last 
decade, with now a 30-day mortality rate of only $2.4 \%$ for ASA III-IV patients aged $71-80$, and $4.3 \%$ for ASA III-IV patients aged $>80$ [2].

Despite the improvements in perioperative care, a treatment that avoids major surgery remains of high interest for older patients. The most commonly used alternative treatment option consists of radiotherapy, with or without local excision. The role of radiotherapy and the different treatment schedules and delivery methods have been described in this special EJSO issue by Sun Myint and Gerard [3]. The most optimal outcome of radiotherapy is a complete or a very good response of the tumor with the patient monitored in a watch and wait protocol. The watch and wait protocol for clinically complete responders was championed and further developed by Habr-Gama and caught worldwide attention with the good results published in 2004 [4]. The approach initially drew a lot of criticism from the surgical community, but with other series corroborating the initial findings, the watch and wait approach has become a valid alternative to major surgery in patients with a clinical complete response. The data and outcome on the largest series of 880 patients was presented by the International Watch and Wait Data registry in 2018 [5]. It showed a regrowth rate of $25 \%$, with the majority located in the bowel wall and occurring in the first two years. The 5-year overall survival was $85 \%$, with many patients dying from unrelated causes, corresponding with a disease specific survival of $97 \%$. The details of the treatment of the local regrowth were not always available, and it was estimated that the rate of locally unsalvageable disease was $1 \%$ at most.

In The Netherlands there is an ongoing collaborative network since 2004 that focusses on the watch and wait strategy (W\&W), with a database containing detailed information and studies on imaging, oncological outcome and quality of life [6-9]. The aim of the present study is to provide information on the outcome of a watch and wait policy for rectal cancer in older patients in this collaborative Dutch database.

\section{Materials and methods}

Patients

Rectal cancer patients who were diagnosed with a clinical complete response after neoadjuvant treatment between 2004 and 2019 were offered a W\&W program through the national network registration. The majority of patients were prospectively included with an informed consent in two IRB approved studies, registered in clinicaltrials.gov since 2009 (NCT00939666 and NCT02278653). Other patients were retrospectively included in the registry, for whom informed consent was waived by the local institutional review board. Patients who were offered and opted for a W\&W were aware that this approach was an alternative treatment that deviated from the standard guideline treatment of TME resection. Inclusion criteria for the present study and analysis were: 1 ) biopsy proven primary rectal adenocarcinoma without distant metastasis at baseline, 2) neoadjuvant treatment with long course CRT $\left(28 \times 1.8 \mathrm{~Gy}\right.$ with $2 \times 825 \mathrm{mg} / \mathrm{m}^{3}$ capecitabine) or short course radiotherapy $(5 \times 5 \mathrm{~Gy})$ and a prolonged waiting interval, 3$) \mathrm{mini}-$ mum age of 75 years or older, 4 ) minimum of 2 years follow-up in a $\mathrm{W} \& \mathrm{~W}$ program, and 5) (near) complete response at first or second response evaluation.

\section{Response assessment}

The response was evaluated $8-12$ weeks after the end of radiotherapy with digital rectal examination, flexible sigmoidoscopy and standard MRI with additional diffusion weighted imaging (MRI-DWI). Criteria for a clinical complete response have been described previously, and consist of no lesions felt at digital rectal examination (DRE), a typical white scar with or without telangiectasia at endoscopy and no signs of residual disease on MRI [6]. Patients with a very good but not a complete response were labelled 'near-complete responses', consisting of superficial ulceration or irregular persisting erythema on endoscopy and intermediate/low residual signal on T2W-MRI and/or small foci of diffusion restriction on MRI-DWI. These patients were offered a second reassessment, $8-12$ weeks after the first evaluation, and could be included in the study.

\section{Follow-up}

The standard follow-up for rectal cancer consisted of computed tomography scan (CT) and carcinoembryogenic (CEA) antigen measurements as recommended by national guidelines every year for 5 years. W\&W patients were additionally followed with a DRE, endoscopy and MRI (+DWI) every 3 months the first year, and 6monthly thereafter up to 5 years.

\section{Functional outcomes}

As a part of the second prospective study (NCT02278653) pelvic functional outcomes were assessed with questionnaires. Defecation problems were assessed with the Vaizey score and the low anterior resection syndrome (LARS) score $[10,11]$. The Vaizey score is a faecal incontinence score based on defecation pattern of the previous 4 weeks and consists of questions regarding frequency, consistency of stools lost and effect on lifestyle. The range of the score is $0-24$ in which a score of 12 or higher is indicated with major incontinence. The LARS-score is a score intended for patients who underwent low anterior resection for rectal cancer, and consists of 5 questions regarding frequency, clustering, urgency and incontinence for flatus or liquid stools. The range of this score is $0-42$ and is divided into no, minor or major LARS (0-20 points; 21-29 points; $30-42$ points respectively). Colostomy-free rate served also as a measure of quality of life. Urinary problems were assessed with the International Prostate Symptom Score (IPSS) [12]. Although this score was designed to assess bladder function in patients with benign prostate hypertrophy, it was judged to be the most suited for the purpose when no other and more focussed questionnaires were available. It consists of 7 questions addressing frequency, urgency, intermittency, weak stream, nocturnia, straining, incomplete bladder emptying and quality of life. The IPSS ranges from 0 to 35 and is divided into mild (0-7), moderate [8-19] and severe symptoms (20-35).

\section{Statistical analysis}

Baseline characteristics were provided. Local regrowth free rate (LRFR), colostomy-free rate (CFR), non-regrowth disease-free survival (NRDFS) and overall survival (OS) were estimated with Kaplan-Meier curves. Duration of follow-up was calculated between end of last CRT and time to event of interest or last follow-up date. Local regrowth was defined as luminal regrowth or involvement of loco regional lymph nodes and local control was defined as the absence of local regrowth. NRDFS was defined as the absence of pelvic failure (local recurrence after delayed TME for local regrowth), the absence of distant metastasis or absence of death. Overall survival was defined as the absence of death. Statistical analyses were performed using the Statistical Package for the Social Sciences (SPSS version 25.0, Inc., Chicago, IL). 
Table 1

Baseline characteristics of all patients. Data are \% $(\mathrm{n} / \mathrm{N})$ unless otherwise stated. $\mathrm{CRT}=$ chemoradiation, Gy = Gray.

\begin{tabular}{ll}
\hline & Total cohort $(\mathrm{N}=43)$ \\
\hline Age, median (range), years & $78(75-87)$ \\
\hline Sex (male) & $67 \%(29 / 43)$ \\
\hline Clinical T stage & \\
\hline T1 & $2 \%(1 / 43)$ \\
T2 & $16 \%(7 / 43)$ \\
T3 & $67 \%(29 / 43)$ \\
T4 & $14 \%(6 / 43)$ \\
\hline Clinical N stage (N+) & $61 \%(26 / 43)$ \\
Distance anal verge (cm) & \\
$<5$ & $71 \%(31 / 43)$ \\
$>5$ & $29 \%(12 / 43)$ \\
\hline Adjuvant chemotherapy & $17 \%(7 / 43)$ \\
\hline Neo-adjuvant therapy & \\
CRT & $93 \%(40 / 43)$ \\
$5 x 5$ Gy & $5 \%(2 / 43)$ \\
Other & $2 \%(1 / 43)$ \\
\hline Timing inclusion & \\
Immediate & $44 \%(19 / 43)$ \\
Reassessment & $56 \%(24 / 43)$ \\
\hline Follow-up time, median (range), months & $37(24-109)$ \\
\hline
\end{tabular}

\section{Results}

\section{Patient characteristics}

Between 2004 and 2019 a total of 304 rectal cancer patients followed a W\&W approach with a minimum follow-up of two years. 43/304 (14\%) patients were 75 years or older and constitute the group of patients for the present study. The majority of these patients were included prospectively $(\mathrm{n}=27)$. Median age was 78 years (range 75-87) and 29/43 (67\%) patients were male. The majority of patients (40/43) received neoadjuvant CRT, and 7/43 (17\%) of patients received adjuvant chemotherapy after CRT. Nineteen of 43 patients (44\%) were included immediately 8-12 weeks after CRT and the remaining 24/43 (56\%) patients were included at reassessment $8-12$ weeks after the first evaluation. Median FU was 37 (24-109) months. See Table 1 for detailed baseline characteristics.

\section{Oncological outcomes}

Five of 43 patients (12\%) developed a local regrowth, all of which were luminal and within two years (range 5-18). Four were detected both on MRI and endoscopy and one was diagnosed only with endoscopy. Three patients underwent a TME resection, one low anterior resection (LAR) and 2 abdominoperineal resection (APR). The remaining two patients underwent local excision. One patient had a local recurrence after TME, leading to an overall pelvic control in $42 / 43$ patients (98\%).

The local regrowth free rate, non-regrowth disease-free survival and overall survival at 3 -years was $88 \%, 91 \%$ and $97 \%$ respectively (see Fig. 1). Three patients developed distant metastasis (1 lung; 1 lung and liver; 1 peritoneal metastasis) and as mentioned above there was one patient with a local recurrence. Four patients eventually died of the following causes: metastatic disease (one patient), other cancer with no evidence or rectal cancer recurrence (2 patients), and a ruptured suprarenal aneurysm (one patient).

\section{Functional outcomes}

Only three of 43 patients ended up with a stoma ( 2 after APR for a regrowth, and one after LAR due to persistent incontinence), resulting in a 3-year colostomy-free rate of 93\% (see Fig. 1). Thirteen patients were included in the prospective study mainly focussing on functional problems (NTC02278653), of whom 9/13 (69\%) completed the Vaizey score and LARS-score, and 7/13 (54\%) completed the IPSS. All responders had a sustained complete response during follow-up.

The mean Vaizey score after 3,12 and 24 months was 4.4 (SD 3.5), 4.2 (SD 4.2) and 5.4 (SD 5.1) respectively, indicating a good continence. Two out of 7 patients (29\%) had major incontinence (score $\geq 12$ ) at 24 months. The mean LARS-score at 3,12 and 24 months was 19.7 (SD 11.1), 19.6 (SD 12.3) and 21.3 (SD 9.4) respectively. Two patients had major LARS (score 30-42) during all time-points (3,12 and 24 months). See Fig. 2 for detailed results. The mean IPSS was 7.3 (SD 6.5) at 3 months, 9.1 (SD 5.9) at 12 months and 9 (SD 5) at 24 months. Only one (14\%) of 7 patients had severe urinary problems after 3 months. See Fig. 3 for detailed results.

\section{Discussion}

The outcome of older patients with a complete or near complete response after radiotherapy for rectal cancer who are followed in a watch and wait protocol in the Dutch national cohort series is very good. The 3-year overall survival is $97 \%$, with death mostly due to other causes. The 3 -year local regrowth rate is $12 \%$, with delayed surgery resulting in a high pelvic control rate of $98 \%$. There was a 3year colostomy-free rate of $93 \%$, and although the sample size of the functional outcome assessment was too small for a reliable analysis, the results suggest a reasonably good anorectal and urinary function.

The overall and disease specific survival is similar to that described in the largest series of 880 patients in the International Watch and Wait Data registry in 2018 [5]. The 25\% 2-year regrowth rate in that series was much higher than in the current study. The baseline tumour characteristics in the two studies were similar, with the majority a T3 tumour with positive nodes, consistent with the accepted indication for neoadjuvant radiotherapy. The difference in regrowth rate most likely reflects the difference in the selection criteria for a complete response, and whether or not a second reassessment is performed for 'near complete responses' before taking a decision. The Dutch collaborative network generally uses a strict definition of complete response, and is liberal with second assessments before deciding on taking patients to surgery or start a W\&W approach, leading to the relatively low regrowth rate when compared to other centres. There is a general concern that omitting TME surgery exposes patients to an oncological risk by uncontrolled pelvic disease and metastatic disease originating from the regrowth. The IWWD data suggest that with a good follow up program the risk for uncontrolled pelvic disease after $\mathrm{W} \& \mathrm{~W}$ for complete responders is $1 \%$ at most, in line with the current findings. The excess metastatic risk in W\&W is more difficult to estimate but the very high disease specific 5-year survival of $95 \%$ suggests that the risk of metastases is more related to tumour biology than to the omission of immediate surgery [5]. For the frail and older patient this oncological risk is more easily counterbalanced by an increased operative risk and a decreased life expectancy than for a younger patient. With a decision-analytic model Smith et al. even suggested an improved survival for $\mathrm{W} \& \mathrm{~W}$ compared to TME surgery for increasing age and comorbidity [13].

The patients in the current study were 75 or older, but the fact that all patients with regrowth were treated surgically and the high 

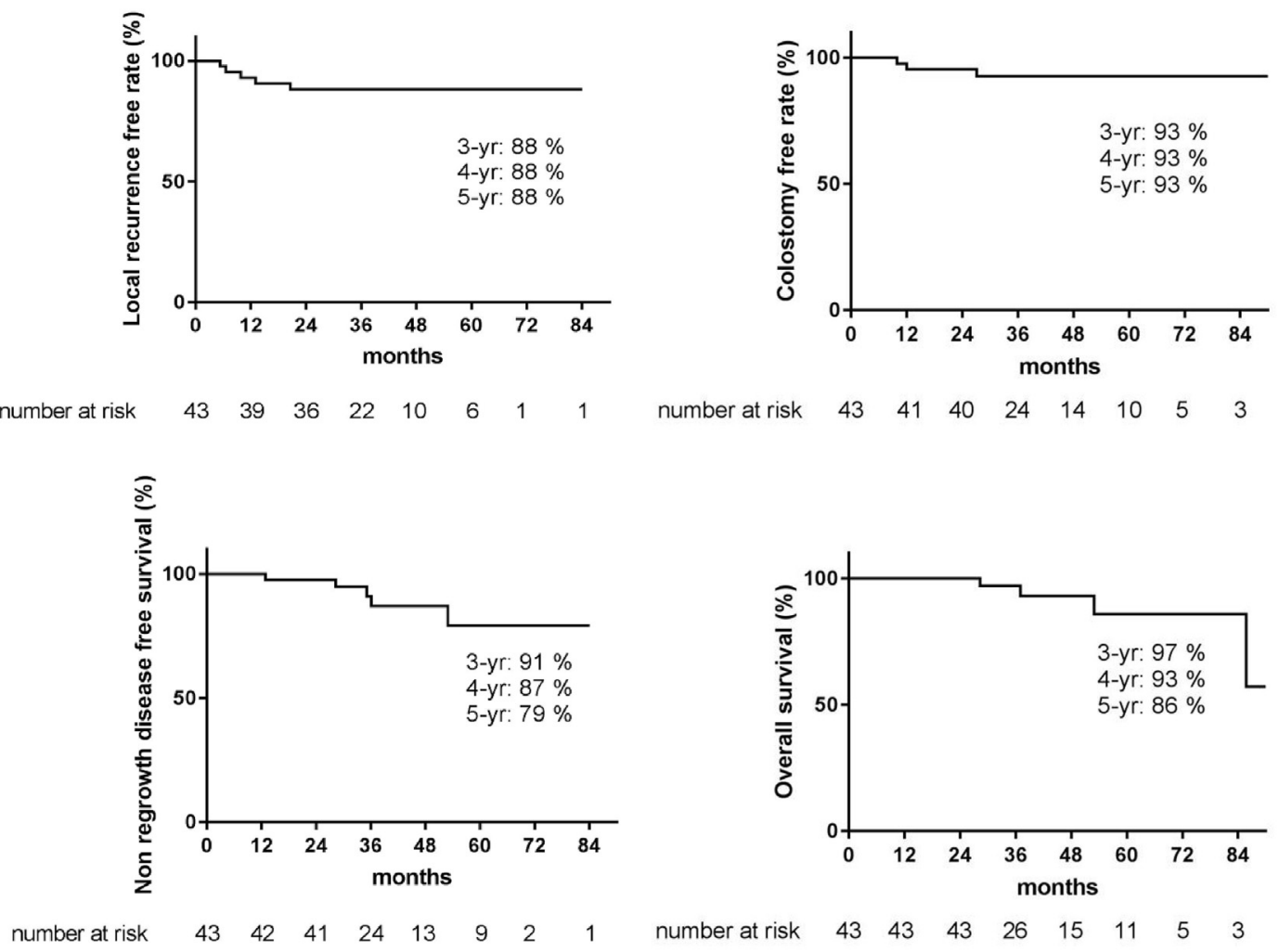

Fig. 1. Kaplan Meier survival curves (local regrowth free rate, non-regrowth disease free survival, overall survival and colostomy-free rate) for all patients.

overall survival suggests that the majority of patients was reasonable fit, and underwent neoadjuvant radiotherapy with the intent to have a TME resection. The very old and frail patients who cannot tolerate major rectal surgery and are treated with radiotherapy as a definitive treatment are underrepresented in the present study, as patients are only registered when they have a complete clinical response at the time of response assessment. Standard neoadjuvant radiotherapy results in complete response in only 15-30\%, depending on the size and T-stage of the tumour [14]. When the explicit goal is to obtain higher local control rates with radiotherapy there are a number of options to deliver a higher dose, with internal or external boost techniques, as reviewed by Sun Myint et al. and Bujko et al. [3,15] Another approach that is expected to yield higher response rates is to add systemic therapy either as induction or consolidation therapy in combination with radiotherapy, but the added toxicity will limit the use in older patients [16]. Major rectal surgery can also be avoided by performing a transanal local excision of a small tumour remnant when there was a good response after radiotherapy [17].

In addition to the benefit of avoiding postoperative complications and mortality major surgery, older patients have a high interest in maintaining a high quality of life and remaining as independent from caretakers as possible. In the current study $71 \%$ of patients presented with a tumour within $5 \mathrm{~cm}$ form the anal verge. For most patients, standard TME surgery would have resulted in a permanent colostomy, or a poor function in a very low anastomosis after neoadjuvant therapy [18]. The 3-year colostomyfree rate was $93 \%$, with only the three patients requiring delayed TME surgery resulting in a permanent colostomy. This is in line with the $95 \%$ reported by Martens et al. and the crude overall $90 \%$ by Smith et al. $[6,19]$ The paper on the UK OnCoRe project by Renehan et al. describes a lower 74\% 3-year colostomy-free survival,
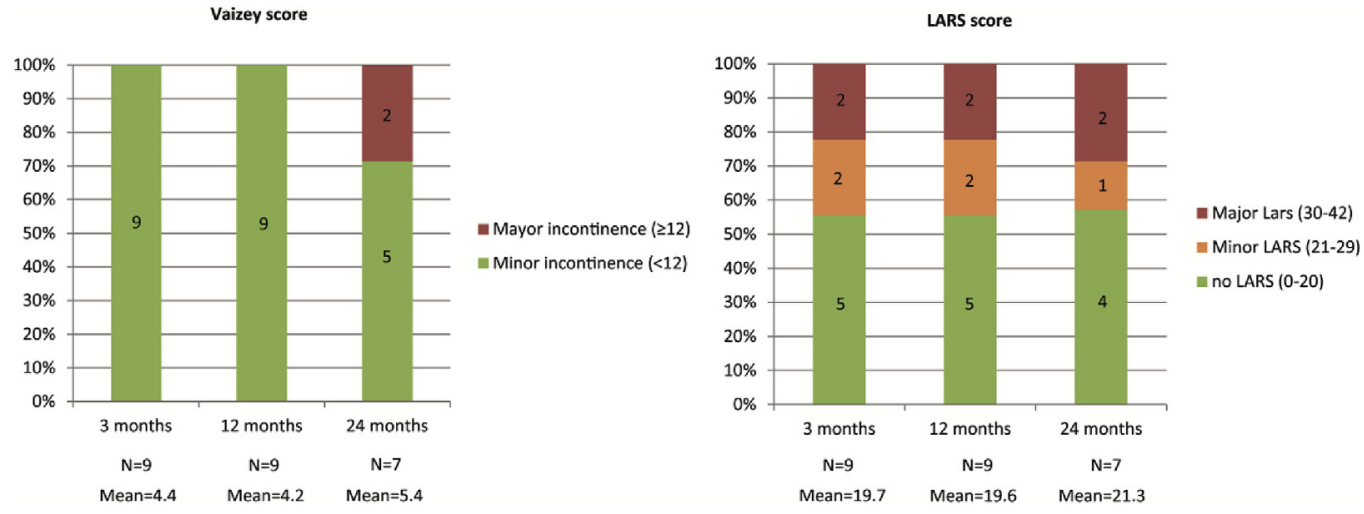

Fig. 2. Defecation problems according the Vaizey- and low anterior resection syndrome (LARS) -score. 
IPSS

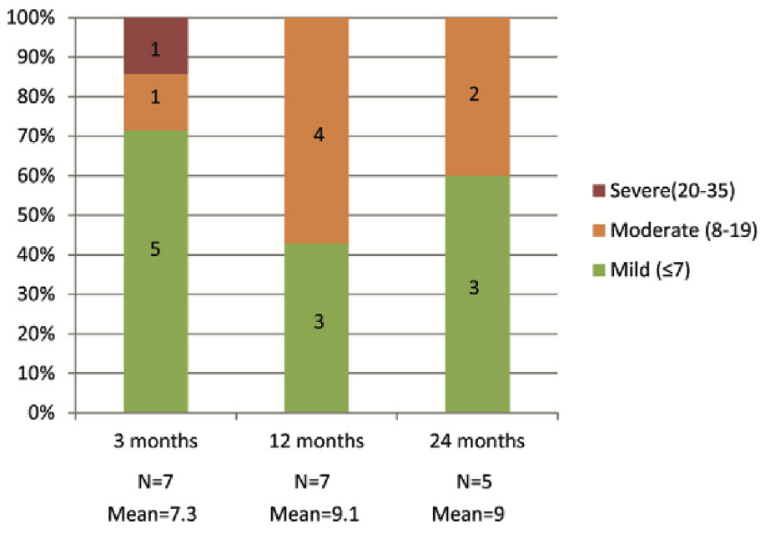

Fig. 3. Urinary problems according International Prostate Symptom Score (IPSS).

related to the broader inclusion and the resulting higher 38\% 3-year local regrowth rate [20]. Radiotherapy by itself has some negative effect on anorectal function, and up to one third of patients in a W\&W program are reporting major LARS symptoms [9]. Although in the current study the number of available questionnaires was too low for a reliable analysis, the results suggest a reasonably good anorectal and urinary function in the majority of patients. The most commonly reported symptoms are clustering and urgency, and in our experience almost all patients can handle this without much interference with daily activities. The majority of patients are very satisfied to have avoided the short and long-term side effects of major surgery and a permanent colostomy.

There are some limitations of the present study. Because of the design of the registry there is an underrepresentation of very old and frail patients who are treated with radiotherapy as a definitive treatment. The encouraging outcome of the W\&W approach in clinical complete responses in reasonable fit older patients in the current study can in our view be extrapolated to the more frail patients, who have even more to gain from avoiding major surgery. A further limitation is the very small number of data on quality of life. Given the importance of this outcome measure in this group of patients, more data should be generated in preferably prospective studies.

\section{Conclusion}

The outcome of older patients with a complete or near complete response after radiotherapy for rectal cancer who are followed in a watch and wait protocol is very good. The pelvic control rate is very high, and the majority of deaths are not related to rectal cancer. Most patients can avoid major surgery and a definitive colostomy, and have a reasonable anorectal and urinary function.

\section{Declaration of competing interest}

The authors declare that they have no known competing financial interests or personal relationships that could have appeared to influence the work reported in this paper.

\section{References}

[1] Rostoft S. Basic geriatric principles for colorectal surgeons: how to optimize assessment and care of the elderly in the perioperative period? Eur J Surg Oncol 2020.

[2] de Neree Tot Babberich MPM, Detering R, Dekker JWT, Elferink MA, Tollenaar R, Wouters M, et al. Achievements in colorectal cancer care during 8 years of auditing in the Netherlands. Eur J Surg Oncol 2018;44(9):1361-70.

[3] Sun Myint A, Gerard JP. Role of radiotherapy in the treatment of rectal cancer in older patient. Eur J Surg Oncol 2020.

[4] Habr-Gama A, Perez RO, Nadalin W, Sabbaga J, Ribeiro Jr U, Silva e Sousa Jr AH, et al. Operative versus nonoperative treatment for stage 0 distal rectal cancer following chemoradiation therapy: long-term results. Ann Surg 2004;240(4): 711-7. discussion 7-8.

[5] van der Valk MJM, Hilling DE, Bastiaannet E, Meershoek-Klein Kranenbarg E, Beets GL, Figueiredo NL, et al. Long-term outcomes of clinical complete responders after neoadjuvant treatment for rectal cancer in the Internationa Watch \& Wait Database (IWWD): an international multicentre registry study. Lancet 2018;391(10139):2537-45

[6] Martens MH, Maas M, Heijnen LA, Lambregts DM, Leijtens JW, Stassen LP, et al. Long-term outcome of an organ preservation program after neoadjuvant treatment for rectal cancer. J Natl Cancer Inst 2016;108(12).

[7] Maas M, Lambregts DM, Nelemans PJ, Heijnen LA, Martens MH, Leijtens JW, et al. Assessment of clinical complete response after chemoradiation for rectal cancer with digital rectal examination, endoscopy, and MRI: selection for organ-saving treatment. Ann Surg Oncol 2015;22(12):3873-80.

[8] Lambregts DMJ, Maas M, Boellaard TN, Pizzi AD, van der Sande ME, Hupkens BJP, et al. Long-term imaging characteristics of clinical complete responders during watch-and-wait for rectal cancer-an evaluation of over 1500 MRIs. Eur Radiol 2019;30(1):272-80.

[9] Hupkens BJP, Martens MH, Stoot JH, Berbee M, Melenhorst J, Beets-Tan RG, et al. Quality of life in rectal cancer patients after chemoradiation: watch-andwait policy versus standard resection - a matched-controlled study. Dis Colon Rectum 2017;60(10):1032-40.

[10] Vaizey CJ, Carapeti E, Cahill JA, Kamm MA. Prospective comparison of faecal incontinence grading systems. Gut 1999;44(1):77-80.

[11] Emmertsen KJ, Laurberg S. Low anterior resection syndrome score: development and validation of a symptom-based scoring system for bowel dysfunction after low anterior resection for rectal cancer. Ann Surg 2012;255(5): 922-8.

[12] Barry MJ, Fowler Jr FJ, O'Leary MP, Bruskewitz RC, Holtgrewe HL, Mebust WK et al. The American urological association symptom index for benign prostatic hyperplasia. The measurement committee of the American urological association. J Urol 1992;148(5):1549-57. discussion 64.

[13] Smith FM, Rao C, Oliva Perez R, Bujko K, Athanasiou T, Habr-Gama A, et al. Avoiding radical surgery improves early survival in elderly patients with rectal cancer, demonstrating complete clinical response after neoadjuvant therapy: results of a decision-analytic model. Dis Colon Rectum 2015;58(2): $159-71$.

[14] Maas M, Nelemans PJ, Valentini V, Das P, Rodel C, Kuo LJ, et al. Long-term outcome in patients with a pathological complete response after chemoradiation for rectal cancer: a pooled analysis of individual patient data. Lancet Oncol 2010;11(9):835-44.

[15] Bujko K, Glynne-Jones R, Papamichael D, Rutten HJT. Optimal management of localized rectal cancer in older patients. J Geriatr Oncol 2018;9(6):696-704.

[16] Petrelli F, Trevisan F, Cabiddu M, Sgroi G, Bruschieri L, Rausa E, et al. Total neoadjuvant therapy in rectal cancer: a systematic review and meta-analysis of treatment outcomes. Ann Surg 2019.

[17] Arezzo A, Lo Secco G, Passera R, Esposito L, Guerrieri M, Ortenzi M, et al. Individual participant data pooled-analysis of risk factors for recurrence after neoadjuvant radiotherapy and transanal local excision of rectal cancer: the PARTTLE study. Tech Coloproctol 2019;23(9):831-42.

[18] Battersby NJ, Bouliotis G, Emmertsen KJ, Juul T, Glynne-Jones R, Branagan G et al. Development and external validation of a nomogram and online tool to predict bowel dysfunction following restorative rectal cancer resection: the POLARS score. Gut 2018;67(4):688-96.

[19] Smith JJ, Strombom P, Chow OS, Roxburgh CS, Lynn P, Eaton A, et al. Assessment of a watch-and-wait strategy for rectal cancer in patients with a complete response after neoadjuvant therapy. JAMA Oncol 2019:e185896.

[20] Renehan AG, Malcomson L, Emsley R, Gollins S, Maw A, Myint AS, et al Watch-and-wait approach versus surgical resection after chemoradiotherapy for patients with rectal cancer (the OnCoRe project): a propensity-score matched cohort analysis. Lancet Oncol 2016;17(2):174-83.

\section{Acknowledgements}

No acknowledgements. 